\title{
Comparação quantitativa da comunidade de aves de um fragmento de floresta semidecidual do interior do Estado de São Paulo em intervalo de 30 anos
}

\author{
Vagner Cavarzere ${ }^{1}$, Rafael S. Marcondes ${ }^{1}$, Gabriel P. Moraes² \& Reginaldo J. Donatelli² \\ 1. Pós-Graduação em Zoologia, Departamento de Zoologia, Instituto de Biociências, Universidade de São Paulo, Rua do Matão, travessa 14, 101, 05508-900 São Paulo, SP. Seção de Aves, \\ Museu de Zoologia da Universidade de São Paulo, Av. Nazaré 481, 04218-970 São Paulo, SP. (cavarzere@usp.br; raf.marcondes@gmail.com) \\ 2. Departamento de Ciências Biológicas, Universidade Estadual Paulista,Av. Engenheiro LuizEdmundo Carrijo Coube 14-01,17033-360 Bauru,SP.(g moraes@hotmail.com;rjdonat@fc.unesp.br)
}

\begin{abstract}
A 30-year quantitative comparison of the bird community of a semideciduous forest remnant in the state of São Paulo. Few studies have evaluated long-term changes in avian abundance in forest remnants. To compare both species richness and abundance of the bird community in a forest fragment located in the municipality of Gália, state of São Paulo, southeastern Brazil, we surveyed forest birds using transect counts. We compared our results with a survey conducted 30 years earlier at the same locality and further classified bird species according to their food habits to eventually predict fluctuations of specific abundance. Although species with population declines predominated in the community, all trophic categories had species which increased their abundances. Most species prone to move around remnants decreased in abundance. We suggest that, regarding specific abundances, trophic categories may be equally affected as a result of fragmentation processes and that the forest regeneration of this remnant may have led to the loss of edge species. Species that suffered from abundance loss during this time period may become locally extinct in the near future.
\end{abstract}

KEYWORDS. Caetetus Ecological Station, fragmentation, species abundance, transect counts, trophic categories.

RESUMO. Poucos estudos avaliaram em longo prazo a variação no tamanho populacional das espécies de aves em fragmentos florestais. Para avaliar a riqueza e a abundância específica da comunidade de aves de um remanescente de mata semidecidual do interior do Estado de São Paulo, sudeste do Brasil, foi conduzido o censo da avifauna florestal utilizando-se a metodologia de contagem em transecção. Estes resultados foram comparados com levantamento realizado na mesma localidade 30 anos antes, e as aves foram classificadas de acordo com suas categorias alimentares com a finalidade de associá-las à tendência ao aumento/diminuição de suas abundâncias após este intervalo de tempo. Embora tenha havido predominância de espécies com diminuição populacional, todas as categorias tróficas analisadas apresentaram também espécies com aumento em suas abundâncias. A maioria das espécies com propensão a deslocarem-se entre fragmentos apresentou diminuição em suas abundâncias. Sugerimos que, em relação a suas abundâncias específicas, as categorias tróficas são igualmente afetadas pelos processos da fragmentação, e que a regeneração florestal sofrida pelo remanescente pode ter resultado na perda de espécies de bordas. Espécies cujas abundâncias tenham reduzido neste intervalo de tempo podem sofrer extinção local futuramente.

PALAVRAS-CHAVE. Abundância de aves, categorias tróficas, contagem em transecção, Estação Ecológica dos Caetetus, fragmentação.

A perda e o declínio de espécies de aves tropicais aumentaram de maneira rápida no final do século passado, principalmente em decorrência do aumento do desmatamento (Willis, 1979; Lovejoy et al., 1986; KatTAN et al., 1994). Os fragmentos resultantes dessa prática variam em tamanho, formato e grau de isolamento de outros remanescentes e acabam com um subconjunto alterado e empobrecido da comunidade biótica original (TURNER, 1996). Mesmo protegidos, fragmentos florestais continuam sofrendo degradação ambiental devido ao seu isolamento, o que compromete a sobrevivência de espécies da avifauna sensíveis a perturbações e, consequentemente, reduz sua biodiversidade (MACARTHUR \& Wilson, 1967). Muitos estudos analisaram em detalhe a variação na riqueza de espécies de aves após a fragmentação florestal (e.g. BierregaArd \& Lovejoy, 1989; Kattan et al., 1994; Aleixo \& Vielliard, 1995; Stouffer \& Bierregaard, 1995; Robinson, 1999; Ferraz et al., 2003; Stouffer et al., 2009). No entanto, poucos são os estudos que avaliaram em longo prazo a variação no tamanho populacional de espécies de aves em fragmentos florestais no Neotrópico (e.g. Antunes, 2005; Anjos et al., 2011). Tal tipo de avaliação pode ser útil para estimar o risco futuro de extinção local de espécies em fragmentos (RoBINSON, 2001).
Alguns autores demonstraram que espécies de aves com dieta específica, tais como insetívoros ou frugívoros, tendem a ser mais sensíveis à redução de habitats florestais (WILLIS, 1979; GRAY et al., 2007; O'DeA \& WhitTAKER, 2007), e a ausência de determinadas espécies desses grupos em ambientes modificados pode ser considerada uma das consequências dos processos de fragmentação. Desse modo, este estudo objetivou quantificar a riqueza de aves em um fragmento de floresta estacional semidecidual no Estado de São Paulo, sudeste do Brasil. Para avaliar a variação na abundância de indivíduos das espécies presentes no fragmento, os resultados registrados nesse levantamento qualiquantitativo foram comparados com aqueles obtidos por levantamento similar realizado há 30 anos (WILLIS \& ONIKI, 1981).

\section{MATERIAL E MÉTODOS}

O estudo foi realizado na Estação Ecológica dos Caetetus (EECa; 22²4'27'S 49¹6'06”W), anteriormente conhecida como Fazenda Paraíso, com aproximadamente 2.180 ha de floresta estacional semidecidual e um dos mais importantes remanescentes desse tipo de vegetação no Estado de São Paulo (Evans et al., 2005; CAVARZERE et al., 2009a). A Estação está 
localizada no limite norte da bacia hidrográfica do médio Rio Paranapanema, entre os municípios de Gália e Alvinlândia, região centro-oeste do Estado de São Paulo (Fig. 1). O clima da região é classificado de acordo com o sistema Köppen no tipo Cwa, mesotérmico de inverno seco, com chuvas de outubro a março e períodos de seca entre abril e setembro. Com altitudes variando de 500 a $680 \mathrm{~m}$, a região possui precipitação e temperatura anuais médias de $1.431 \mathrm{~mm} \mathrm{e} 21,5^{\circ} \mathrm{C}$, respectivamente (TABANEZ et al., 2005).

$\mathrm{O}$ mapeamento da vegetação da EECa indicou 12 unidades fisionômicas diferenciadas segundo o porte, densidade, condições de preservação e condições ecológicas. A vegetação mais próxima do clímax apresenta componente arbóreo denso em ótimo estado de preservação e alta densidade em todos os estratos, ao passo que espécies pioneiras, lianas e bambus são ausentes. Essa fisionomia é encontrada apenas no interior do fragmento. No outro extremo encontra-se o estrato arbóreo baixo e esparso, com alto índice de perturbação, predominando espécies pioneiras, lianas, bambus e sub-bosque abundante e diversificado. Essa vegetação predomina ao redor do remanescente (TABANEZ et al., 2005). São comuns nos estratos vegetais Metrodorea nigra St. Hil., Savia dictyocarpa Müll. Arg., Ocotea indecora (Schott) Meins., Trichilia catigua A. Juss e Aspidosperma polyneuron Müll. Arg., com alturas variando de 8 a $32 \mathrm{~m}$ (DuRIGAN et al., 2000). O ambiente matriz da EECa é composto por pastos e plantações de café e, mais recentemente, soja (obs. pess.), enquanto os grandes fragmentos de mata mais próximos encontramse a pelo menos $1 \mathrm{~km}$ de distância (TABANEZ et al., 2005).

Coleta de dados. O método de contagem em transecção (BiBBy et al., 2000) foi empregado durante o período matutino na amostragem da avifauna para as análises aqui apresentadas. Buscou-se com essa escolha a comparação entre este estudo e aquele conduzido por Willis \& ONIKI (1981) na EECa 30 anos antes, o qual utilizou transecções para a contagem de espécies e indivíduos. Foram percorridas as trilhas existentes no interior do fragmento, sempre a uma distância mínima de $100 \mathrm{~m}$ das bordas. As trilhas eram percorridas ao longo de $3 \mathrm{~h}$ durante duas manhãs consecutivas, aproximadamente $10 \mathrm{~min}$ antes do nascer do sol. As amostragens mensais ocorreram ao longo de 14 meses, entre novembro de 2005 e dezembro de 2006. Nas trilhas onde foram realizadas as amostragens há predomínio de vegetação arbórea de porte alto e denso com pouca ou nenhuma perturbação de caráter antrópico (TABANEZ et al., 2005).

A velocidade das caminhadas foi constante $(1,5$ $\mathrm{km} / \mathrm{h}$ ) e o sentido percorrido das transecções, sempre o mesmo. O raio de detecção foi considerado ilimitado, sendo as amostragens realizadas pela mesma equipe de observadores (VC e GPM). As aves foram identificadas visualmente com auxílio de binóculos 8 × 20 e 8 × 42 e por meio de suas vocalizações, registradas com gravador
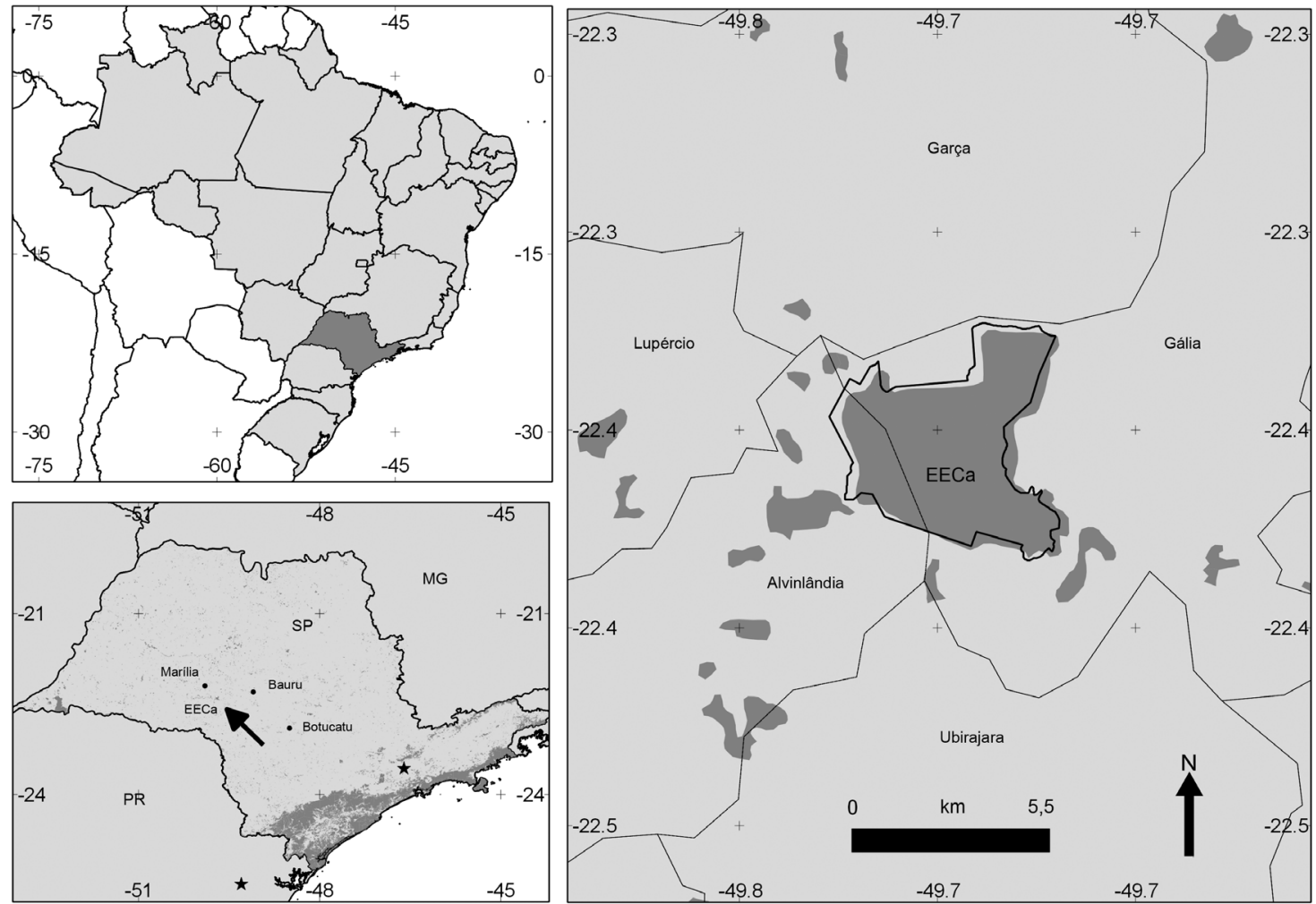

Fig. 1. Localização da Estação Ecológica dos Caetetus (EECa), região centro-oeste do Estado de São Paulo, sudeste do Brasil. Estrelas representam capitais estaduais e a seta indica a localização do fragmento da EECa entre os municípios de Gália e Alvinlândia. Os remanescentes florestais do Estado de São Paulo estão representados em cinza-escuro. 
cassete (microfone embutido). Atenção especial foi dada para não contar o mesmo indivíduo mais de uma vez. Evitou-se realizar transecções durante períodos de chuva ou ventos fortes. As gravações encontramse depositadas no Arquivo Sonoro da Seção de Aves do Museu de Zoologia da Universidade de São Paulo (MZUSP).

Análises. De modo a avaliar quantitativamente a composição da comunidade, para cada espécie registrada foi obtido um número de contatos, o qual foi ajustado proporcionalmente para cada $100 \mathrm{~h}$ de observações de modo a evitar abundâncias com casas decimais (Willis \& ONIKI, 1981). As aves foram classificadas segundo hábitos alimentares e estratos preferenciais de forrageamento de acordo com bibliografia específica (Willis, 1979) e observações adicionais em campo. Também segundo Willis (1979), as espécies foram classificadas como propensas ou não a se deslocar entre fragmentos florestais. O status de abundância específica adotado seguiu o seguinte modelo: muito comuns (MC; $>100$ registros $/ 100 \mathrm{~h}$ de observações ao longo dos meses de amostragem), comuns (C;100 $\geq 50$ registros), pouco comuns (PC, $49 \geq 15$ registros), incomuns (I, $14 \geq$ 5 registros) e raras ( $\mathrm{R} ;<5$ registros). Espécies endêmicas da Mata Atlântica seguem PARKER et al. (1996).

A curva cumulativa de espécies e cálculos de estimadores não paramétricos de riqueza (Chao1 e Jack1) foram realizados com base em 1.000 randomizações da ordem das amostras mensais com o programa EstimateS 8.2 (Colwell, 2009). Comparações entre abundâncias específicas durante tempos diferentes $(\mathrm{T} 1=$ WiLlis \& ONIKI [1981], T2 = este estudo) foram realizadas com teste de chi-quadrado (goodness of fit), cuja hipótese nula considerou as espécies igualmente abundantes nos dois tempos. Apenas espécies com um mínimo de 30 indivíduos, ao menos em um dos tempos, foram analisadas. $\mathrm{O}$ nível de significância adotado foi $\alpha=0,05$. O Índice de Similaridade de Chao-Jaccard, baseado em incidência e abundância das espécies (CHAO et al., 2005) foi utilizado para a análise da similaridade da comunidade florestal de aves entre os dois tempos considerados. A sequência taxonômica e os nomes dos táxons seguem as resoluções do Comitê Brasileiro de Registros Ornitológicos (CBRO, 2011).

\section{RESULTADOS}

Foram registradas 116 espécies de aves e 2.862 contatos em aproximadamente 84 horas de transecções. Apenas espécies florestais foram consideradas, sendo descartadas as características de ambientes abertos ouvidas dentro da mata e também as vistas sobrevoando o fragmento, pois sua utilização por estas espécies não pode ser confirmada. Desse modo, o número de espécies utilizado para as análises foi de 100 e a abundância total, de 2.795 indivíduos. A curva de acumulação de espécies mostrou que o aumento do esforço amostral

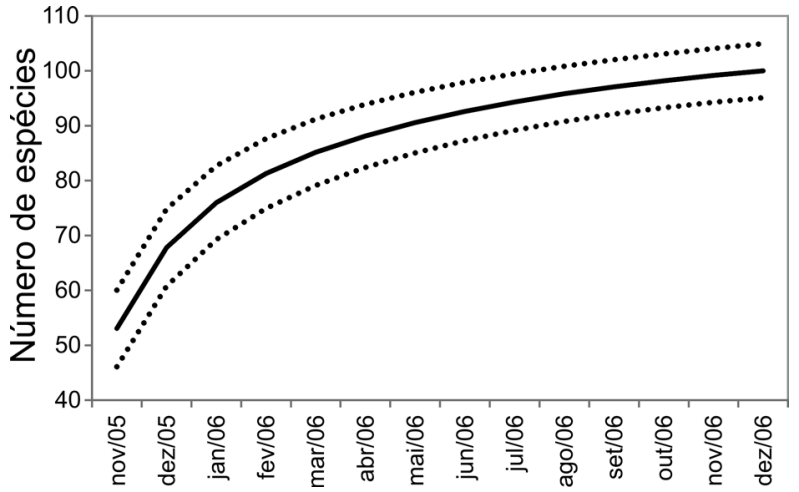

Fig. 2. Curva de acumulação (linha sólida) e respectivos intervalos de $95 \%$ de confiança (linhas pontilhadas) das espécies registradas no fragmento de mata da Estação Ecológica dos Caetetus, Gália, São Paulo, entre novembro de 2005 e dezembro de 2006. A ordem das amostras foi randomizada 1.000 vezes e a curva representa os valores médios obtidos para cada mês.

deve acrescentar poucas espécies florestais devido à tendência de estabilização a partir dos últimos meses de amostragem (Fig. 2), fato também sugerido pelos estimadores de riqueza não paramétricos Chao1 e Jack1, com 105 e 111 espécies estimadas, respectivamente.

WILLIS \& ONIKI (1981) registraram 141 espécies de aves, mas seguindo-se os critérios de análise das espécies aqui descritos, 98 foram consideradas estritamente florestais. Somando-se os dois estudos, o total de espécies florestais registradas para a EECa foi de 118, sendo 18 exclusivas de T1 e 20 exclusivas de T2. Das 18 espécies exclusivas de T1, seis são endêmicas da Mata Atlântica e apenas uma espécie, Pyrrhura frontalis (Vieillot, 1817), havia sido considerada comum em T1. Em relação às 20 espécies presentes em $\mathrm{T} 2$ e ausentes em T1, nenhuma obteve registros suficientes para enquadrá-las nas categorias de comum ou muito comum; quatro são endêmicas da Mata Atlântica (Tab. I). A similaridade da avifauna entre os dois períodos foi de 75,6\%, com 80 espécies compartilhadas (Tabs I-III); 21 espécies não apresentaram valores de abundância suficientes para análises (Tab. II). Com base na abundância específica reportada durante T1 foi possível avaliar a flutuação da abundância de algumas espécies presentes nos dois estudos. Das 59 espécies com abundância suficiente para análises, 42 demonstraram variação significativa de abundância em intervalo de 30 anos (chi-quadrado, $P<0,05)$. Destas, $13(31 \%)$ apresentaram valores maiores de abundância (incluindo seis endêmicas da Mata Atlântica), enquanto 29 (69\%) tiveram redução em suas abundâncias (11 endêmicas). Adicionalmente, duas espécies, Dysithamnus mentalis (Temminck, 1823) e Cyclarhis gujanensis (Gmelin, 1789), apresentaram redução marginalmente significativa de suas abundâncias (Tab. III).

Das 42 espécies que tiveram variação significativa na abundância, $10(23 \%)$ são frugívoras de grande porte, 19 (43\%) insetívoras com diferentes tipos de especialização, $12(27 \%)$ onívoras e uma (2\%) granívora. Das frugívoras, $40 \%$ tiveram aumento na abundância e $60 \%$ tiveram diminuição. Para as 
Tab. I. Espécies registradas exclusivamente durante contagens em transecções na Estação Ecológica dos Caetetus, Gália, São Paulo, no estudo de WiLLIS \& ONIKI (1981; T1) e neste estudo (T2). São indicadas categorias alimentares, categorias de forrageamento, abundância a cada $100 \mathrm{~h}$ de observações e o status de presença das espécies (R, raras; C, comuns; PC, pouco comuns; I, incomuns). Endemismos de Mata Atlântica (MA) seguem PARKER et al. (1996). Espécies separadas por serem propensas (P) ou não propensas (N) a deslocarem-se entre fragmentos. Asteriscos indicam espécies cuja ocorrência foi documentada por meio de gravações.

\begin{tabular}{|c|c|c|c|c|c|}
\hline & & Categoria trófica & Abundância & Status & MA \\
\hline & \multicolumn{5}{|l|}{ Espécies T1 } \\
\hline \multirow{10}{*}{$\mathrm{N}$} & Micrastur ruficollis & Carnívoro diurno & 2 & $\mathrm{R}$ & \\
\hline & Pyrrhura frontalis & Frugívoro (frutos grandes) & 77 & $\mathrm{C}$ & $\mathrm{x}$ \\
\hline & Pteroglossus bailloni & Frugívoro (frutos grandes) & 2 & $\mathrm{R}$ & $\mathrm{x}$ \\
\hline & Terenura maculata & Insetívoro de bambu ou emaranhados & 5 & I & $\mathrm{x}$ \\
\hline & Malacoptila striata & Insetívoro de sub-bosque (artrópodes grandes) & 26 & $\mathrm{PC}$ & $\mathrm{x}$ \\
\hline & Nonnula rubecula & Insetívoro de sub-bosque (artrópodes grandes) & 5 & I & \\
\hline & Lochmias nematura & Insetívoro de sub-bosque (artrópodes grandes) & 4 & $\mathrm{R}$ & \\
\hline & Melanerpes flavifrons & Insetívoro de troncos e galhos & 44 & $\mathrm{PC}$ & $\mathrm{x}$ \\
\hline & Tityra inquisitor & Onívoro (frutos e artrópodes grandes) & 7 & I & \\
\hline & Cissopis leverianus & Onívoro de borda & 31 & $\mathrm{PC}$ & \\
\hline \multirow{8}{*}{$\mathrm{P}$} & Capsiempis flaveola & Insetívoro de borda & 7 & I & \\
\hline & Contopus cinereus & Insetívoro de meia-altura & 4 & $\mathrm{R}$ & \\
\hline & Phylloscartes ventralis & Insetívoro de sub-bosque (artrópodes pequenos na folhagem) & 7 & I & \\
\hline & Veniliornis spilogaster & Insetívoro de troncos e galhos & 9 & I & $\mathrm{x}$ \\
\hline & Tityra cayana & Onívoro (frutos e artrópodes grandes) & 15 & I & \\
\hline & Cacicus haemorrhous & Onívoro de borda & 9 & I & \\
\hline & Pachyramphus polychopterus & Onívoro de copa & 15 & I & \\
\hline & Nemosia pileata & Pequeno onívoro de copa & 18 & $\mathrm{PC}$ & \\
\hline \multirow{9}{*}{$\mathrm{N}$} & Espécies T2 & & & & \\
\hline & Micrastur semitorquatus* & Carnívoro diurno & 4 & $\mathrm{R}$ & \\
\hline & Hylocharis cyanus & Comedor de néctar e insetos & 1 & $\mathrm{R}$ & \\
\hline & Pteroglossus aracari & Frugívoro (frutos grandes) & 6 & I & \\
\hline & Taraba major* & Insetívoro de sub-bosque (artrópodes grandes) & 1 & $\mathrm{R}$ & \\
\hline & Thamnophilus pelzelni* & Insetívoro de sub-bosque (artrópodes pequenos na folhagem) & 10 & I & \\
\hline & Campephilus robustus & Insetívoro de troncos e galhos & 2 & $\mathrm{R}$ & $\mathrm{x}$ \\
\hline & Oxyruncus cristatus & Pequeno onívoro de copa & 5 & I & \\
\hline & Turdus albicollis* & Pequeno onívoro de sub-bosque & 4 & $\mathrm{R}$ & \\
\hline \multirow{12}{*}{$\mathrm{P}$} & Coereba flaveola* & Comedor de néctar e insetos & 26 & $\mathrm{PC}$ & \\
\hline & Florisuga fusca & Comedor de néctar e insetos & 5 & I & $\mathrm{x}$ \\
\hline & Phaethornis eurynome & Comedor de néctar e insetos & 4 & $\mathrm{R}$ & $\mathrm{x}$ \\
\hline & Brotogeris chiriri* & Frugívoro (frutos grandes) & 36 & $\mathrm{PC}$ & \\
\hline & Patagioenas cayennensis & Frugívoro (frutos grandes) & 20 & $\mathrm{PC}$ & \\
\hline & Leptotila rufaxilla & Grande frugívoro de solo & 21 & $\mathrm{PC}$ & \\
\hline & Haplospiza unicolor* & Granívoro de borda & 1 & $\mathrm{R}$ & $\mathrm{x}$ \\
\hline & Colaptes melanochloros* & Insetívoro de troncos e galhos & 5 & I & \\
\hline & Turdus leucomelas* & Onívoro de borda & 35 & $\mathrm{PC}$ & \\
\hline & Tangara cayana & Onívoro de borda & 5 & I & \\
\hline & Euphonia chlorotica* & Pequeno onívoro de copa & 17 & $\mathrm{PC}$ & \\
\hline & Turdus subalaris & Pequeno onívoro de sub-bosque & 4 & $\mathrm{R}$ & \\
\hline
\end{tabular}

insetívoras, $31 \%$ tiveram aumento e $69 \%$ tiveram redução, e para as onívoras, os valores foram $25 \%$ e $75 \%$, respectivamente. A única espécie granívora demonstrou redução em sua abundância. Ainda, das 42 espécies com variação significativa na abundância, 15 (34\%) foram consideradas propensas a se deslocar entre fragmentos. Destas, quatro (26\%) apresentaram aumento na abundância e 11 (73\%) apresentaram diminuição. As 27 espécies restantes foram consideradas não propensas a se deslocar entre fragmentos e, destas, nove (33\%) apresentaram aumento e 18 (66\%) apresentaram diminuição em suas abundâncias (Tab. III). 
Tab. II. Espécies que não obtiveram abundância necessária para análises estatísticas e registradas durante contagens em transecções na Estação Ecológica dos Caetetus, Gália, São Paulo, no estudo de WiLlis \& ONIKI (1981; T1) e neste estudo (T2). São indicadas categorias alimentares, categorias de forrageamento e o status de presença das espécies (R, raras; C, comuns; PC, pouco comuns; I, incomuns). Endemismos de Mata Atlântica (MA) seguem PARKER et al. (1996). Espécies separadas por serem propensas (P) ou não propensas (N) a deslocarem-se entre fragmentos. Mig = espécie migratória. Asteriscos indicam espécies cuja ocorrência foi documentada por meio de gravações.

\begin{tabular}{|c|c|c|c|c|c|}
\hline & Espécie & Categoria trófica & Status T1 & Status T2 & MA \\
\hline \multirow{12}{*}{$\mathrm{N}$} & Thalurania glaucopis & Comedor de néctar e insetos & I & I & $\mathrm{x}$ \\
\hline & Geotrygon montana* & Grande frugívoro de solo & $\mathrm{R}$ & I & \\
\hline & Drymophila ochropyga & Insetívoro de bambu ou emaranhados & I & I & $\mathrm{x}$ \\
\hline & Hemitriccus diops* & Insetívoro de bambu ou emaranhados & $\mathrm{PC}$ & $\mathrm{PC}$ & $\mathrm{x}$ \\
\hline & Xiphocolaptes albicollis & Insetívoro de sub-bosque (artrópodes grandes) & $\mathrm{PC}$ & $\mathrm{R}$ & \\
\hline & Dendrocolaptes platyrostris* & Insetívoro de sub-bosque (artrópodes grandes) & $\mathrm{PC}$ & $\mathrm{PC}$ & \\
\hline & Sclerurus scansor* & Insetívoro de sub-bosque (artrópodes grandes) & $\mathrm{PC}$ & I & $\mathrm{x}$ \\
\hline & Dendrocincla turdina & Insetívoro de sub-bosque (artrópodes grandes) & $\mathrm{R}$ & I & $\mathrm{x}$ \\
\hline & Basileuterus flaveolus* & Insetívoro de sub-bosque (artrópodes pequenos) & I & $\mathrm{PC}$ & \\
\hline & Celeus flavescens & Insetívoro de troncos e galhos & I & I & \\
\hline & Nyctiphrynus ocellatus & Insetívoro noturno & I & $\mathrm{R}$ & \\
\hline & Euphonia violacea & Pequeno onívoro de copa & $\mathrm{PC}$ & $\mathrm{R}$ & \\
\hline \multirow{9}{*}{$\mathrm{P}$} & Poecilotriccus plumbeiceps* & Insetívoro de bambu ou emaranhados & I & I & \\
\hline & Cnemotriccus fuscatus & Insetívoro de borda & I & $\mathrm{R}$ & \\
\hline & Aramides saracura & Insetívoro de sub-bosque (artrópodes grandes) & $\mathrm{PC}$ & $\mathrm{R}$ & $\mathrm{x}$ \\
\hline & Veniliornis passerinus* & Insetívoro de troncos e galhos & I & I & \\
\hline & Dryocopus lineatus & Insetívoro de troncos e galhos & I & $\mathrm{R}$ & \\
\hline & Lurocalis semitorquatus $^{\text {mig* }^{*}}$ & Insetívoro noturno & I & $\mathrm{PC}$ & \\
\hline & Saltator similis* & Onívoro de borda & $\mathrm{PC}$ & I & \\
\hline & Dacnis cayana & Pequeno onívoro de copa & I & I & \\
\hline & Antilophia galeata* & Pequeno onívoro de sub-bosque & $\mathrm{R}$ & $\mathrm{R}$ & \\
\hline
\end{tabular}

Tab. III. Resultado do teste de chi-quadrado $\left(\chi^{2}\right)$ das espécies com aumento (+) ou diminuição (-) de suas abundâncias e registradas durante contagens em transecções na Estação Ecológica dos Caetetus, Gália, São Paulo, no estudo de WiLlis \& ONIKI (1981; T1) e neste estudo (T2). Espécies em negrito são aquelas com valores de abundância significativamente diferentes. São indicadas categorias alimentares, categorias de forrageamento, abundância a cada $100 \mathrm{~h}$ de observações e o status de presença das espécies (R, raras; C, comuns; PC, pouco comuns; I, incomuns; MC, muito comuns). Endemismos de Mata Atlântica (MA) seguem PARKER et al. (1996). Espécies separadas por serem propensas (P) ou não propensas $(\mathrm{N})$ a deslocarem-se entre fragmentos. A letra "m" indica resultado marginalmente significativo. Mig = espécie migratória. Asteriscos indicam espécies cuja ocorrência foi documentada por meio de gravações.

\begin{tabular}{|c|c|c|c|c|c|c|c|c|c|c|}
\hline & Espécie & Categoria trófica & $\mathrm{T} 1$ & $\mathrm{~T} 2$ & $\chi^{2}$ & $P$ & Relação & $\begin{array}{c}\text { Status } \\
\mathrm{T} 1\end{array}$ & $\begin{array}{l}\text { Status } \\
\mathrm{T} 2\end{array}$ & MA \\
\hline \multirow{13}{*}{$\mathrm{N}$} & Penelope superciliaris & Frugívoro (frutos grandes) & 126 & 5 & 112,4 & 0,000 & - & MC & I & \\
\hline & Odontophorus capueira* & Grande frugívoro de solo & 119 & 13 & 84,9 & 0,000 & - & MC & I & $\mathrm{x}$ \\
\hline & Cyanocorax chrysops & $\begin{array}{c}\text { Onívoro } \\
\text { (frutos e artrópodes grandes) }\end{array}$ & 106 & 12 & 75,1 & 0,000 & - & $\mathrm{MC}$ & I & \\
\hline & Mackenziaena severa & $\begin{array}{l}\text { Insetívoro de bambu ou } \\
\text { emaranhados }\end{array}$ & 85 & 10 & 60,3 & 0,000 & - & $\mathrm{C}$ & $\mathrm{I}$ & $\mathrm{x}$ \\
\hline & Myiopagis caniceps & Insetívoro de copa & 62 & 1 & 58,5 & 0,000 & - & $\mathrm{C}$ & $\mathrm{R}$ & \\
\hline & Lanio melanops & Pequeno onívoro de sub-bosque & 91 & 14 & 55,9 & 0,000 & - & $\mathrm{C}$ & I & $\mathrm{x}$ \\
\hline & Xiphorhynchus fuscus* & Insetívoro de troncos e galhos & 16 & 87 & 48,9 & 0,000 & + & PC & $\mathrm{C}$ & $\mathrm{x}$ \\
\hline & Hemitriccus orbitatus & $\begin{array}{c}\text { Insetívoro de sub-bosque } \\
\text { (artrópodes pequenos na folhagem) }\end{array}$ & 42 & 5 & 29,7 & 0,000 & - & $\mathrm{PC}$ & I & $\mathrm{x}$ \\
\hline & Myiornis auricularis* & $\begin{array}{l}\text { Insetívoro de bambu ou } \\
\text { emaranhados }\end{array}$ & 31 & 1 & 27,6 & 0,000 & - & PC & $\mathrm{R}$ & $\mathrm{x}$ \\
\hline & Philydor lichtensteini* & Insetívoro de meia-altura & 62 & 18 & 24,4 & 0,000 & - & $\mathrm{C}$ & PC & $\mathrm{x}$ \\
\hline & Leptopogon amaurocephalus* & $\begin{array}{c}\text { Insetívoro de sub-bosque } \\
\text { (artrópodes pequenos na folhagem) }\end{array}$ & 75 & 29 & 20,8 & 0,000 & - & $\mathrm{C}$ & PC & \\
\hline & Trogon surrucura* & $\begin{array}{c}\text { Onívoro } \\
\text { (frutos e artrópodes grandes) }\end{array}$ & 49 & 99 & 16,8 & 0,000 & + & PC & $\mathrm{C}$ & $\mathrm{x}$ \\
\hline & Schiffornis virescens & Pequeno onívoro de sub-bosque & 69 & 29 & 16,8 & $\mathbf{0 , 0 0 0}$ & - & C & PC & $\mathrm{x}$ \\
\hline
\end{tabular}


Tab. III. (Cont.)

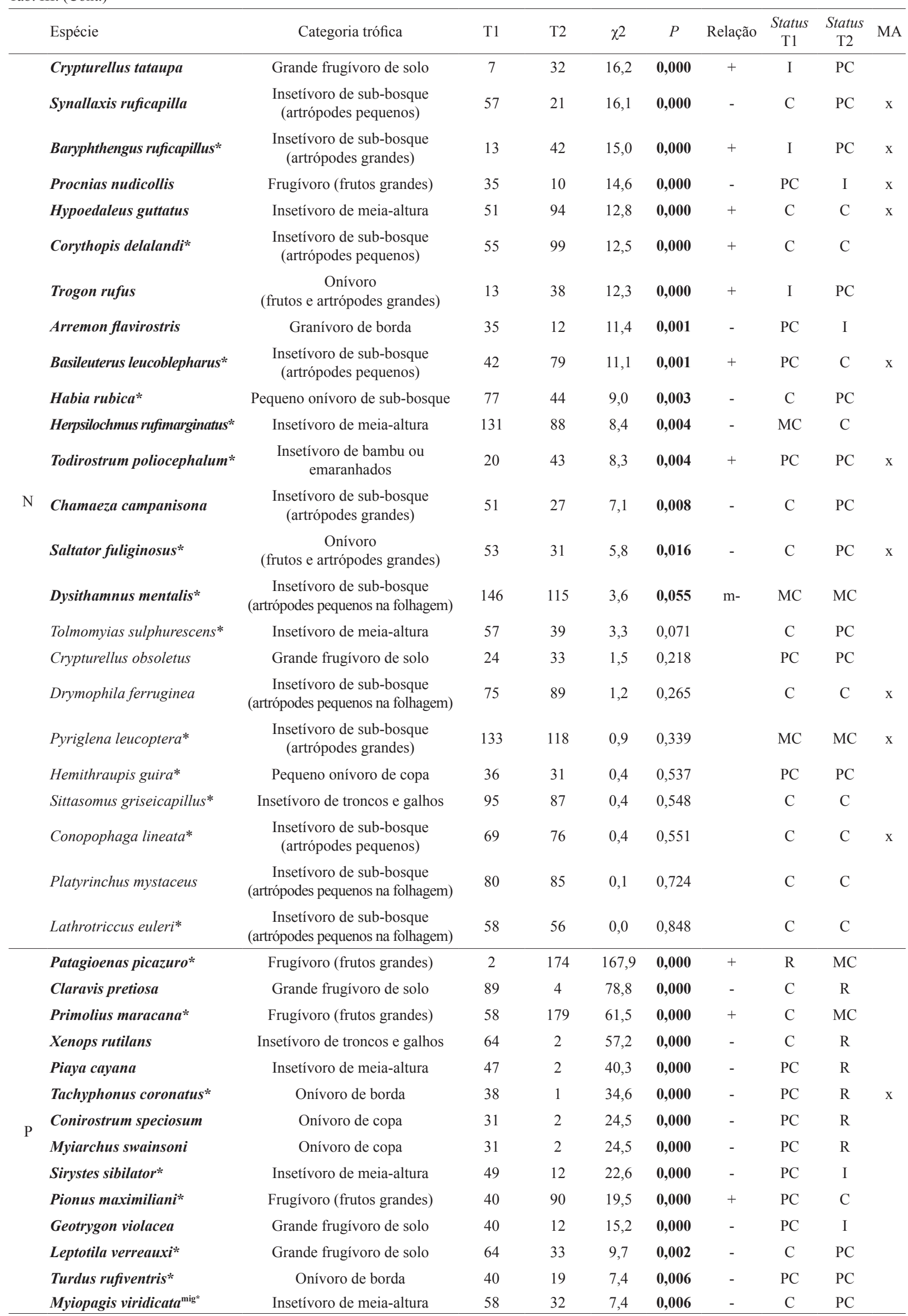




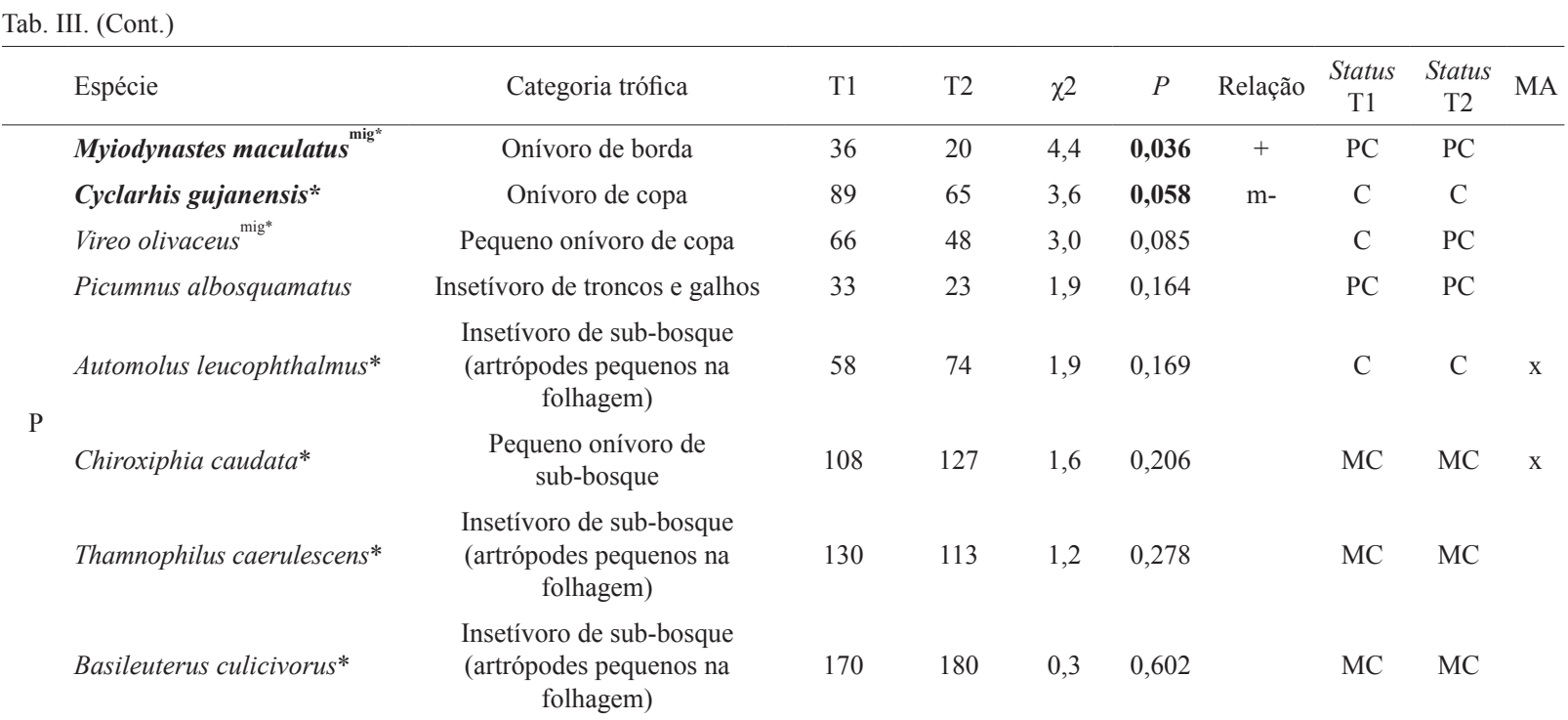

\section{DISCUSSÃO}

Todas as categorias tróficas analisadas apresentaram espécies com aumento e diminuição em suas abundâncias no intervalo considerado de 30 anos, não havendo padrão claro que sustente a afirmação de que espécies com maior especificidade alimentar tenham sofrido unicamente perda de indivíduos em suas populações. Embora tenha havido predominância de espécies com declínio populacional, outras, dentro das mesmas categorias tróficas, parecem ter-se beneficiado (aumento populacional) com a fragmentação. Insetívoros efrugívoros são as categorias alimentares mais abundantes e com maior número de espécies em matas tropicais preservadas (O'DeA \& WhitTAKer, 2007), mas o fato de apenas uma espécie florestal carnívora e seis espécies florestais nectarívoras terem sido registradas no presente estudo não representou artefato amostral ou ausência de espécies devido à fragmentação. Embora aves de rapina estejam entre as mais afetadas com a descaracterização do ambiente (RIBON et al., 2003), estudos conduzidos em florestas estacionais semideciduais no Estado de São Paulo também registraram riqueza próxima à encontrada na EECa (Willis, 1979; Aleixo \& Vielliard, 1995; Donatelli et al., 2004, 2007; Antunes, 2005). Assim, o número de espécies nestas categorias alimentares só pode ser considerado baixo quando comparado à riqueza de insetívoros ou frugívoros. Adicionalmente, outra espécie carnívora, Micrastur ruficollis (Vieillot, 1817), foi registrada exclusivamente em levantamento qualitativo (fora da contagem durante transecções), sendo descartada das análises quantitativas.

Devido à curva cumulativa das espécies ter mostrado tendência à estabilização logo no sétimo mês de amostragem, e ao fato dos valores de riqueza estimados terem-se aproximado da riqueza observada, é possível concluir que a maioria das espécies florestais existentes no fragmento foi registrada no presente levantamento (Colwell \& Coddington, 1994; Chao et al., 2009). Apesar de diversas espécies terem sido registradas apenas durante um dos tempos considerados, é difícil afirmar com segurança se o fragmento perdeu alguma espécie. A ausência em T2 de Pyrrhura frontalis (Vieillot, 1817), frugívora de grande porte comum em $\mathrm{T} 1$, sugere que a espécie tenha sido substituída por Brotogeris chiriri (Vieillot, 1818), também frugívora de grande porte e típica de ambientes mais secos do interior do Brasil, não registrada por WILLIS \& ONIKI (1981). Uma espécie sobre a qual se pode ter certa segurança sobre a extinção no fragmento é Pteroglossus bailloni (Vieillot, 1819). Durante T1, WILlIS \& ONIKI (1981) registraram apenas um indivíduo da espécie na EECa e nenhum outro foi registrado desde então. Por se locomover ampla e conspicuamente em busca de alimento, essa espécie deveria ter sido registrada, caso presente. Pyrrhura frontalis e Pteroglossus bailloni são, também, endêmicas da Mata Atlântica. O endemismo é uma das categorias típicas entre as mais suscetíveis à extinção local como resultado da fragmentação de habitats (Terborgh \& Winter, 1980; Kattan et al., 1994; Christiansen \& Pitter, 1997; Anjos et al., 2010).

Diversos estudos documentaram a perda de espécies em áreas fragmentadas da Região Neotropical (e.g. RiBon et al., 2003; Anjos et al., 2010 e referências), mas poucos (ANTUNEs, 2005; AnJos et al., 2011) discutiram a variação na abundância das espécies presentes, importante indicador do risco futuro de extinção local (RoBInson, 2001). Espécies são perdidas continuamente e, antes disso, têm suas abundâncias reduzidas por até diversas décadas após a fragmentação inicial (KatTAn et al., 1994; RoBinson, 1999; SigeL et al., 2006; Stouffer et al., 2009). Antunes (2005) reportou o aumento da abundância relativa de 70 espécies e diminuição de outras 36, considerando aves florestais e de áreas abertas, no município de Anhembi, São Paulo, em intervalo entre inventários de 25 anos. No presente estudo, 29 espécies florestais tiveram diminuição na abundância, enquanto 13 tiveram 
aumento. Apesar de o número total de espécies florestais na EECa ter permanecido praticamente estável e com grande similaridade da composição das espécies, esse resultado indica que futuramente diversas espécies podem se extinguir localmente, diminuindo a riqueza total do fragmento. Espécies como Myiornis auricularis (Vieillot, 1818) e Myiopagis caniceps (Swainson, 1835) estão entre as que tiveram maior redução na abundância e agora são raras no fragmento, correndo risco de extinção local (ver abaixo sobre espécies de borda).

Estudos relataram que espécies frugívoras de grande porte e espécies insetívoras, especialmente aquelas que forrageiam diretamente ou próximo ao solo, são as mais afetadas pela fragmentação do habitat (Willis, 1979; KatTan et al., 1994; StOUfFer \& BierRegaARD, 1995; Robinson, 1999; Gray et al., 2007; Stouffer et al., 2009). Aqui se observou que a maioria das espécies com maior especificidade alimentar apresentou redução em suas abundâncias $(60 \%$ das frugívoras e $68 \%$ das insetívoras), confirmando sua vulnerabilidade à fragmentação. É possível que a perda de espécies insetívoras de sub-bosque esteja relacionada à ausência de micro-habitats especializados no fragmento (KATTAN et al., 1994), ou aos grandes territórios de forrageamento requeridos por tais espécies, principalmente aquelas que forrageiam em bandos mistos (Develey \& Stouffer, 2001). No entanto, os mecanismos que levam às respostas específicas das diferentes guildas à fragmentação ainda são pouco entendidos e necessitam de mais investigação (Gray et al., 1997). Um resultado importante do presente estudo foi a forte tendência de redução na abundância das espécies onívoras (75\% das espécies), proporcionalmente maior que a redução na abundância das espécies com dieta mais específica. Tal resultado é inesperado uma vez que espécies onívoras geralmente são menos afetadas (RiBON et al., 2003) ou até aumentam de abundância após a fragmentação (Sigel et al., 2006).

A regeneração florestal no fragmento pode fornecer uma explicação para essas observações. Ao menos desde 1976, quando a EECa passou à propriedade do Estado (TABANEZ et al., 2005), não houve mais perturbação florestal na área por fatores antrópicos (Evans et al., 2005) e desde então o fragmento passa por processo de regeneração. Pode-se supor que atualmente a Estação apresenta características mais próximas ao clímax e menos áreas perturbadas do que entre 1976 e 1979 (Tabarelli \& Mantovani, 1999), quando Willis \& ONIKI (1981) a visitaram. Espécies onívoras estão associadas à degradação do ambiente e aos habitats de borda por serem favorecidas por uma dieta generalista (Willis, 1979; O’Dea \& Whittaker, 2007). Assim, a regeneração florestal, com diminuição na área de habitats com características de borda ou secundárias, pode ter influenciado a redução na abundância de membros dessa categoria alimentar. Poderia se esperar que, com a regeneração no fragmento, espécies de guildas com dieta mais específica, como insetívoros ou frugívoros, tivessem apresentado aumento em suas abundâncias. Isso não ocorreu, possivelmente devido a outros fatores, como o isolamento do fragmento em relação a outros blocos florestais que poderiam fornecer espécies, ou à área insuficiente da Estação. Similarmente, Antunes (2005) demonstrou que 20 espécies florestais e de borda foram perdidas no remanescente por ele estudado no interior de São Paulo, que se encontra protegido contra o desmatamento há mais de 25 anos.

A maturação do habitat também foi indicada como causa de perda de espécies na ilha de Barro Colorado, no Panamá (Robinson, 1999), e na Mata Atlântica do sudeste de São Paulo (Aleixo, 1999). É certo que a regeneração de uma área é acompanhada por uma diminuição dos nichos disponíveis, já que mudanças na estrutura da vegetação produzem áreas de habitats em estádios intermediários de regeneração. Esse padrão de perturbação permite a coexistência de espécies florestais e de borda numa mesma área, levando ao aumento da riqueza e diversidade da comunidade de aves local, em particular, para espécies generalistas (SCHEMSKe \& Brokaw, 1981; Wunderle JR et al., 1987; Johns, 1996). Para as espécies com dieta mais especializada, a maior ou menor abundância pode estar relacionada ao que ocorreu após a fragmentação ou durante a regeneração.

Houve maior proporção de espécies com declínio na abundância entre aquelas com propensão a voar entre fragmentos em relação àquelas sem esta habilidade ( $73 \%$ versus $66 \%$, respectivamente). Tal resultado foi também inesperado já que espécies com capacidade de dispersão e que apresentaram diminuição em suas abundâncias, como Piaya cayana (Linnaeus, 1766), Turdus rufiventris Vieillot, 1818 e Leptotila verreauxi Bonaparte, 1855, são consideradas menos suscetíveis à extinção local, pois a redução na população devido ao tamanho reduzido do fragmento pode ser compensada por imigração (WILlis, 1979; RoBINSON, 1999). A regeneração florestal também pode explicar, ao menos em parte, esse resultado. Tipicamente, espécies que habitam ambientes secundários ou de borda se dispersam bem (ANJos \& Boçon, 1999; RoBinson, 2001). Entre as 11 espécies consideradas propensas a se deslocar entre fragmentos e que tiveram diminuição na abundância no presente estudo, nove são classificadas por PARKER et al. (1996) como habitantes de borda de mata e/ou ambientes em estádios iniciais e intermediários de sucessão vegetal. Portanto, as espécies com alta capacidade de deslocamento entre fragmentos podem, assim como as espécies onívoras, ter sido negativamente afetadas pela regeneração florestal na EECa durante as últimas décadas.

Houve tendência de redução na abundância da maioria das espécies de todas as guildas alimentares desde que WiLlis \& ONIKI (1981) visitaram a área de estudo, independente de a propensão das espécies deslocarem-se entre fragmentos ser alta ou baixa. Parte dessa redução, principalmente para as espécies 
onívoras e propensas à dispersão, pode ser explicada pela regeneração florestal. No entanto, a redução na abundância também atingiu muitas espécies típicas de florestas preservadas, como, por exemplo, Procnias nudicollis (Vieillot, 1817), Odontophorus capueira (Spix, 1825), Lanio melanops (Vieillot, 1818) e Schiffornis virescens (Lafresnaye, 1838), indicando que o fragmento, apesar de sua grande área, não está sendo suficiente para preservá-las, possivelmente devido ao isolamento em relação a uma área maior fornecedora de indivíduos das mesmas espécies (DEVELEy \& MARTENSEN, 2006). Como já apontado por CAVARZERE et al. (2009b), o contínuo monitoramento quali-quantitativo da avifauna da Estação Ecológica dos Caetetus é de extrema importância para o entendimento em longo prazo de como aves sensíveis à fragmentação irão se comportar neste fragmento. Por não haver áreas de floresta contínua próximas à estação, o que possibilitaria a colonização de espécies que já desapareceram ou tiveram redução populacional, a extinção dessas espécies na EECa pode ser irreversível sem projetos de reintrodução. Futuras pesquisas devem se concentrar no entendimento das causas da perda de espécies em vez de meramente catalogar aquelas próximas a desaparecer.

Agradecimentos. Somos gratos à Célia e ao Teodoro pelas conversas e recepções na EECa. Aos amigos Andreli C. Dalbeto, Fernanda G. Maciel, Flávio K. Ubaid e Carolina D. Ferreira, pela companhia durante a realização deste estudo. Dois revisores anônimos fizeram sugestões que contribuíram para a melhora do manuscrito.

\section{REFERÊNCIAS BIBLIOGRÁFICAS}

Aleixo, A. 1999. Effects of selective logging on a bird community in the Brazilian Atlantic Forest. Condor 101(3):537-548.

Aleixo, A. \& Vielliard, J. M. E. 1995. Composição e dinâmica da avifauna da mata de Santa Genebra, Campinas, São Paulo, Brasil. Revista Brasileira de Zoologia 12(3):493-511.

Anjos , L. \& Boçon, R. 1999. Bird communities in natural forest patches in southern Brazil. The Wilson Bulletin 111(3):397-414.

Anjos, L.; Collins, C. D.; Holt, R. D.; Volpato, G. H.; Mendonça, L. B.; Lopes, E. V.; Boçom, R.; Bisheimer, M. V.; Serafini, P. P. \& Carvalho, J. 2011. Bird species abundance-occupancy patterns and sensitivity to forest fragmentation: implications for conservation in the Brazilian Atlantic forest. Biological Conservation 144(9):2213-2222.

Anjos, L.; Holt, R. D. \& Robinson, S. 2010. Position in the distributional range and sensitivity to forest fragmentation in birds: a case history from the Atlantic forest, Brazil. Bird Conservation International 20:392-399.

Antunes, A. Z. 2005. Alterações na composição da comunidade de aves ao longo do tempo em um fragmento florestal no sudeste do Brasil. Ararajuba 13(1):47-61

Bibby, C. J.; Burgess, N. D.; Hill, D. A. \& Mustoe, S. 2000. Bird census techniques. London, Academic Press. 256p.

BierregaArd, R. O. \& Lovejoy, T. E. 1989. Effects of forest fragmentation on Amazonian understory bird communities. Acta Amazonica 19:215-241.

Cavarzere, V.; Moraes, G. P. \& Donatelli, R. J. 2009a. Avifauna da Estação Ecológica dos Caetetus, interior de São Paulo, Brasil. Papéis Avulsos de Zoologia 49(35):477-485.

2009b. Diversidade de aves em uma mata estacional da região centro-oeste de São Paulo, Brasil. Revista Brasileira de Biociências 7(4):364-367.

CBRO - Comitê Brasileiro de Registros Ornitológicos. 2011. Listas das aves do Brasil. 10ed. Disponível em $<\mathrm{http}$ ://www. cbro.org.br>. Acesso em 03.02.2012.
Chao, A.; Chazdon, R.; Colwell, R. K. \& Shen, T. J. 2005. A new statistical approach for assessing similarity of species composition with incidence and abundance data. Ecology Letters 8(1):148159.

Chao, A.; Colwell, R. K.; Lin, C. W. \& Gotelli, N. J. 2009. Sufficient sampling for asymptotic minimum species richness estimators. Ecology 90(4):1125-1133.

Christiansen, M. B. \& Pitter, E. 1997. Species loss in a forest bird community near Lagoa Santa in southeastern Brazil. Biological Conservation 80(1):23-32.

Colwell, R. K. 2009. EstimateS: Statistical estimation of species richness and shared species from samples. Versão 8.2. Disponível em: <http://viceroy.eeb.uconn.edu/EstimateS $>$. Acesso em 15.12.2010.

Colwell, R. K. \& Coddington, J. A. 1994. Estimating terrestrial biodiversity through extrapolation. Philosophical Transactions of the Royal Society (Series B) 345(1311):101-118.

Develey, P. F. \& Martensen, A. C. 2006. As aves da Reserva Florestal do Morro Grande (Cotia, SP). Biota Neotropica 6(2):1-16.

Develey, P. F. \& Stouffer, P. C. 2001. Effects of roads on movements by understory birds in mixed-species flocks in central Amazonian Brazil. Conservation Biology 15(5):1416-1422.

Donatelli, R. J.; Costa, T. V. V. \& Ferreira, C. D. 2004. Dinâmica da avifauna em fragmento de mata na Fazenda Rio Claro, Lençóis Paulista, São Paulo, Brasil. Revista Brasileira de Zoologia 21(1):97-114.

Donatelli, R. J.; Ferreira, C. D.; Dalbeto, A. C. \& Posso, S. 2007. Análise comparativa da assembléia de aves em dois remanescentes florestais no interior do Estado de São Paulo, Brasil. Revista Brasileira de Zoologia 24(2):362-375.

Durigan, G.; Franco, G. A. D. C.; Saito, M. \& Baitello, J. B. 2000. Estrutura e diversidade do componente arbóreo da floresta na Estação Ecológica dos Caetetus, Gália, São Paulo. Revista Brasileira de Botânica 23(4):371-383.

Evans, B. E. I.; Ashley, J. \& Marsden, S. J. 2005. Abundance, habitat use, and movements of Blue-winged Macaws (Primolius maracana) and other parrots in and around an Atlantic forest reserve. Wilson Bulletin 117(2):154-164.

Ferraz, G.; Russell, G. J.; Stouffer, P. C.; Bierregaard, R. O.; Pimm, S. L. \& Lovejoy, T. E. 2003. Rates of species loss from Amazonian forest fragments. Proceeding of the National Academy of Sciences 100(24): 14069-14073.

Gray, M. A.; Baldauf, S. L.; Mayhew, P. J. \& Hill, J. H. 2007. The response of avian feeding guilds to tropical forest disturbance. Conservation Biology 21(1):133-141.

JoHns, A. D. 1996. Bird population persistence in Sabahan logging concessions. Biological Conservation 75(1):3-10.

Kattan, G. H.; Alvarez-Lópes, H. \& Giraldo, M. 1994. Forest fragmentation and bird extinctions: San Antonio eighty years later. Conservation Biology 8(1):138-146.

Lovejoy, T. E.; Bierregaard Jr, R. O.; Rylands, A. B. ; Malcolm, J. R. ; Quintela, C. E.; Herper, L. H.; Brown Jr, K. S.; Powell, A. H.; Powell, G. V. N.; Schubart, H. O. R. \& Hays, M. B. 1986. Edge and other effects of isolation on Amazon forest fragments In: SOULÉ, M. ed. Conservation biology: the science of scarcity and diversity. Sanderland, Sinauer Associates. p.257-285.

Macarthur, R. H. \& Wilson, E. O. 1967. The theory of island biogeography. Princeton, Princeton University Press. 224p.

O’Dea, N. \& Whittaker, R. J. 2007. How resilient are Andean montane forest bird communities to habitat degradation? Biodiversity Conservation 16(4): 1131-1159.

Parker, T. A.; Stotz, D. \& FitzPatrick, J. W. 1996. Ecological and distributional databases. In: Stotz, D.; FitzPatrick, J. W.; PARKer, T. A. \& Moskovits, D. K. eds. Neotropical birds: ecology and conservation. Chicago, University of Chicago Press. p.118-436.

Ribon, R.; Simon, J. E. \& Mattos, G. T. 2003. Bird extinctions in Atlantic forest fragments of the Viçosa region, southeastern Brazil. Conservation Biology 17(6):1827-1839.

Robinson, W. D. 1999. Long-term changes in the avifauna of Barro Colorado Island, Panama, a tropical forest isolate. Conservation Biology 31(1):85-97.

2001. Changes in abundance of birds in a Neotropical forest fragment over 25 years: a review. Animal Biodiversity and Conservation 24(2):51-65.

Schemske, D. W. \& Brokaw, N. 1981. Treefalls and the distribution of understory birds in a tropical forest. Ecology 62(4):938-945. 
Sigel, B. J.; Sherry, T. W. \& Young, B. E. 2006. Avian community response to lowland tropical rainforest isolation: 40 years of change at La Selva Biological Station, Costa Rica. Conservation Biology 20(1):111-121.

Stouffer, P. C. \& Bierregand, R. O. 1995. Use of Amazonian forest fragments by understory insectivorous birds. Ecology 76(8):24292445 .

Stouffer, P. C.; Strong, C. \& Naka, L. N. 2009. Twenty years of understorey bird extinctions from Amazonian rain forest fragments: consistent trends and landscape-mediated dynamics. Diversity and Distributions 15(1):88-97.

Tabarelli, M. \& Mantovani, W. 1999. A regeneração de uma floresta tropical montana após corte e queima (São Paulo-Brasil). Revista Brasileira de Biologia 59(2):239-250.

Tabanez, M. F.; Durigan, G.; Keuroghlian, A.; Barbosa, A. F.; Freitas, C. A.; Silva, C. E. F.; Silva, D. A.; Eaton, D. P.; Brisolla, G.; Faria, H. H.; Mattos, I. F. A.; Lobo, M. T.; Barbosa, M. R.; Rossi, M.; Souza, M. G.; Machado, R. B.; Pfeifer, R. M.; Ramos, V. S.; Andrade, W. J. \& Contieri, W. A. 2005. Plano de manejo da Estação Ecológica dos Caetetus. Instituto Florestal Série Registros 29:1-104.

Terborgh, J. \& Winter, B. 1980. Some causes of extinction. In: Soulé, M. E. \& Wilcox, B. A. eds. Conservation Biology: an evolutionary-ecological perspective. Sunderland, Sinauer Associates. p.119-133.

TURNER, I. M. 1996. Species loss in fragments of tropical rain forest: a review of the evidence. Journal of Applied Ecology 33(2):200209.

WiLlis, E. O. 1979. The composition of avian communities in remanescent woodlots in southern Brazil. Papéis Avulsos de Zoologia 33(1):1-25.

Willis, E. O. \& ONIKI, Y. 1981. Levantamento preliminar de aves em treze áreas do Estado de São Paulo. Revista Brasileira de Biologia 41(1):121-135.

Wunderle JR, J. M.; Diaz, A.; Velazquez, I. \& Scharrón, R. 1987. Forest openings and the distribution of understory birds in a Puerto Rican rainforest. Wilson Bulletin 99(1):22-37. 